Groups Geom. Dyn. 6 (2012), 639-658

DOI $10.4171 / \mathrm{GGD} / 168$
Groups, Geometry, and Dynamics

(C) European Mathematical Society

\title{
On the separation profile of infinite graphs
}

\author{
Itai Benjamini, Oded Schramm*and Ádám Timár
}

\begin{abstract}
Initial steps in the study of inner expansion properties of infinite Cayley graphs and other infinite graphs, such as hyperbolic ones, are taken, in a flavor similar to the well-known Lipton-Tarjan $\sqrt{n}$ separation result for planar graphs. Connections to relaxed versions of quasi-isometries are explored, such as regular and semiregular maps.
\end{abstract}

Mathematics Subject Classification (2010). 05C15, $20 \mathrm{~F} 65$.

Keywords. Separation, quasi-isometry, group property, asymptotic dimension.

\section{Introduction}

The best-known separation type result is the Lipton-Tarjan [16] theorem, claiming that there is a way of removing $O(\sqrt{n})$ vertices (and the edges reaching them) from an $n$-vertex planar graph, so that each of the remaining connected components has at most $n / 2$ vertices (see also [1], [2], [17], [18] for other proofs and generalizations).

Rather than considering finite graphs, we will look at infinite graphs, and consider the separation function. The separation function at $n$ is the supremum over all subgraphs of size $n$, of the number of vertices needed to be removed from the subgraph, in order to cut it to connected pieces of size at most $n / 2$. We are interested in separation functions up to constant factors. See the exact definitions in Section 1.1 below.

The separation function (or profile) is a natural coarse geometric invariant of infinite graphs and path metric spaces. In this work we would like to view the separation function as such an invariant, in the family of invariants like isoperimetric profiles and volume growth.

One question we are interested in is, which separation functions are possible for transitive graphs or Cayley graphs? What is the separation function of a given Cayley graph? E.g., what is it for groups of intermediate growth? Our results below will give examples of Cayley graphs with finite separation (free groups), logarithmic

\footnotetext{
* Oded died while solo climbing Guye Peak in Washington State on September 1, 2008.
} 
separation (planar hyperbolic groups), $n^{(d-1) / d}$-separation, $d \in \mathbb{N}\left(\mathbb{Z}^{d}, \mathbb{H}^{d+1}\right)$, $n / \log n$-separation (product of free groups).

We do not know of any example of a vertex transitive graph with separation $n^{\alpha}$, $0<\alpha<1 / 2$. It is maybe of interest to look at separation function for natural families of groups, e.g., groups generated by finite automata ([19]), lamplighter groups, random groups, Kazhdan groups .... The latter could be natural candidates for groups with linear separation, if there is any.

Question 1.1. Is there linear separation, or $n / \log n$ is the largest growth possible?

In which groups do balls or the minimizers of the isoperimetric problem or convex sets admit the largest separation among all sets of a fixed size?

Understanding separation is useful in figuring out the partial order given by regular maps (see Section 1.1 below) on the collection of all spaces, or rather, on the most familiar ones.

In [5] it was shown that bounded degree transient planar graphs admit non-constant bounded Dirichlet harmonic functions. Gil Kalai asked in 1994 whether square root separation can replace planarity? The answer is negative, see [5].

By a comb we mean the $\mathbb{Z}^{2}$ grid when all the edges parallel to $x$-axis but not on the $x$-axis removed. A comb $\times \mathbb{Z}$ is an example of a transient graph with square root separation that does not admit non-constant bounded harmonic functions (in particular, non-constant harmonic Dirichlet functions), and thus is not planar [5].

Question 1.2. Does spectral radius $<1$ plus an additional separation condition imply the existence of non-constant bounded harmonic functions?

Indeed, finite separation transient graphs admit non-constant bounded harmonic functions: for any such bounded size cut separating the graph to two components if only one component were transient we would get a transient simple random walk escaping to infinity via infinitely many cut sets of bounded size, which is impossible, yet having two transient components connected via a finite set implies the existence of non-constant bounded harmonic functions. What about a weaker separation condition?

In [4] it is shown that if to each level of a binary tree the edges of an expander sequence are added, the result has no non-constant bounded harmonic functions. This explains that some separation condition for Question 1.2 is necessary.

In Section 2 we prove that finite separation is the same as bounded treewidth (see definition there), and we present a theorem about the structure of infinite graphs with finite separation.

Then we study the separation of products of graphs, Section 3. First, a bound on the separation function of a product is given. Later it is shown that for a regular tree $T$,

$$
\operatorname{sep}_{T \times T}(n) \asymp \frac{n}{\log n},
$$


where $\asymp$ means up to constant factor. For functions $f$ and $g$, we write $f=O(g)$ or $g=\Omega(f)$ to denote that there exists a $c>0$ such that $f(x) \leq c g(x)$ for every $x$. In Section 4, on Gromov-hyperbolic graphs (or hyperbolic graphs for short), we open with establishing the separation function for $\mathbb{H}^{d}$ followed by a gap theorem, showing that for hyperbolic graphs the separation function is either a constant, or is growing at least logarithmically.

A regular map between two graphs is a map that increases distances at most by some linear function, and such that there is a uniform bound on the number of preimages of a vertex (see Section 1.1 for the precise definition). Given a regular map, the separators for a constant neighborhood of the image can be pulled back, thus the separation function is monotone non decreasing under regular maps (see Lemma 1.3). Section 5 discusses regular maps.

In the last section it is proved that there is no regular map from $\mathbb{Z}^{2}$ to the $\mathbb{Z}_{2}$ lamplighter over $\mathbb{Z}$. The notion of a semi-regular map is introduced and discussed. Asymptotic dimension is monotone under semi-regular maps, hence this seems to be the right type of function for its study (the way that regular maps are suitable for separation).

Many open problems are scattered along the paper.

\subsection{Some definitions}

Definition. Let $G$ be a graph. Suppose that $S \subset G$ is given. Let $\operatorname{cut}(S)$ denote the infimum of the sizes of subsets $C_{S}$ of $S$, so that the largest connected component of $S-C_{S}$ has size smaller than $|S| / 2$. For such a $C_{S}$ we will say (with a slight sloppiness in terminology) that $C_{S}$ separates $S$.

The separation function $\operatorname{sep}_{G}(x): \mathbb{R} \rightarrow \mathbb{R}$ is

$$
\operatorname{sep}_{G}(x)=\sup _{S \subset G,|S|=x} \operatorname{cut}(S) .
$$

Remark. The separation function can be defined in a wider context of path metric spaces, Riemannian manifolds, in particular. For simplicity of the exposition we stick to graphs. When the separation of a manifold is discussed, the reader can replace the manifold by a rough isometric graph, defined as follows.

Definition. Let $\left(X, d_{X}\right),\left(Y, d_{Y}\right)$ be metric spaces, and let $\kappa<\infty$. A $\kappa$-rough isometry (or $\kappa$-quasi-isometry) $f$ from $X$ to $Y$ is a (not necessarily continuous) map $f: X \rightarrow Y$ such that

$$
\kappa^{-1} d_{X}\left(x_{1}, x_{2}\right)-\kappa \leq d_{Y}\left(f\left(x_{1}\right), f\left(x_{2}\right)\right) \leq \kappa d_{X}\left(x_{1}, x_{2}\right)+\kappa
$$

holds for all $x_{1}, x_{2} \in X$, and for every $y_{1} \in Y$ there is some $x_{1} \in X$ such that

$$
d_{Y}\left(y_{1}, f\left(x_{1}\right)\right) \leq \kappa .
$$

If such an $f$ exists, we say that $X$ and $Y$ are roughly isometric. 
It is straightforward to check that roughly isometric graphs have the same separation. In particular, the separation function is a group-invariant for finitely generated groups (it does not depend on which Cayley graph is chosen). However, there is a coarser equivalence relation that still preserves the separation function. This equivalence relation can be defined by the existence of regular maps between the two graphs in both directions (and graphs will turn out to have monotone increasing separation under regular maps).

Definition. Let $\left(X, d_{X}\right),\left(Y, d_{Y}\right)$ be metric spaces, and let $\kappa<\infty$. A (not necessarily continuous) map $f: X \rightarrow Y$ is $\kappa$-regular if the following two conditions are satisfied.

(1) $d_{Y}\left(f\left(x_{0}\right), f\left(x_{1}\right)\right) \leq \kappa\left(1+d_{X}\left(x_{0}, x_{1}\right)\right)$ holds for every $x_{0}, x_{1} \in X$, and

(2) for every open ball $B=B\left(y_{0}, 1\right)$ with radius 1 in $Y$, the inverse image $f^{-1}(B)$ can be covered by $\kappa$ open balls of radius 1 in $X$.

A regular map is a map which is $\kappa$-regular for some finite $\kappa$. Write $X \rightarrow_{\text {reg }} Y$ if there is a regular map from $X$ to $Y$.

It is easy to check that if there is a rough isometry between bounded degree graphs $X$ and $Y$, then there is a regular map from $X$ to $Y$ (and also to the other direction, by the symmetry of being roughly isometric - which is not apparent from the definition). Hence being roughly isometric implies the existence of regular maps from $X$ to $Y$ and from $Y$ to $X$. However, the existence of a regular map from $X$ to $Y$, and one from $Y$ to $X$, does not imply that $X$ and $Y$ would be rough isometric. For example, consider two copies of $\mathbb{Z}^{2}$ "glued" along the axis $\{(0, n)\}$ for $X$, and $\mathbb{Z}^{2}$ for $Y$; see [5].

Regular maps were studied in a somewhat different context by David and Semmes [10]. One use of the separation function is as an obstruction for the existence of a regular map from one graph to another. This is so because if there is a regular map from $X$ into $Y$, then $\operatorname{cut}_{X}(x) \leq C \operatorname{sep}_{Y}(x)$ for some $C<\infty$.

Let $\operatorname{cut}^{c}(G)$ denote the minimal number of vertices that are necessary to separate the finite graph $\mathrm{G}$ into $c$ times smaller pieces. Let $\operatorname{sep}_{G}^{c}(x):=\sup _{S \subset G,|S| \leq x} \operatorname{cut}^{c}(S)$ (in particular, $\operatorname{sep}_{G}^{1 / 2}(x)=\operatorname{sep}_{G}(x)$ ). Then

$$
\operatorname{sep}_{G}^{c}(x) \asymp \operatorname{sep}_{G}(x) .
$$

To see this, suppose $c<1 / 2$, and consider some graph of size $x$. After we cut it into pieces of size at most half of the original one, we can repeat the procedure for the smaller pieces, and iterating if necessary, we can get to graphs of size at most $c$ times that of the original one. Now, in the constantly many steps of the procedure, each separating set used along the way is bounded by $\operatorname{sep}_{G}^{1 / 2}(x)$, hence their total size is at most a constant times $\operatorname{sep}_{G}^{1 / 2}(x)$. (When $c>1 / 2$, do the same thing, but starting from a $c$-separating set.) 
Lemma 1.3. Let $X$ and $Y$ be graphs with a uniform upper bound $d$ on their degrees, and suppose that there is a $\kappa$-regular map $f$ from $X$ to $Y$. Then $\operatorname{sep}_{X}(x)=$ $O\left(\operatorname{sep}_{Y}(x)\right)$.

Proof. Let $A \subset X$ be an arbitrary set of vertices that induces a connected graph. Define $A^{\prime} \subset Y$ as the $2 \kappa$-neighborhood of $f(A)$. By $\kappa$-regularity, $A^{\prime}$ is connected, and it has size at most $|A| d^{2 \kappa}$. Let $S^{\prime}$ be a minimal subset of $A^{\prime}$ that separates it into pieces $C_{1}^{\prime}, \ldots, C_{m}^{\prime}$, each of size at most $A^{\prime} /\left(2 \kappa d^{2 \kappa}\right)$.

Let $S_{0}$ be the $2 \kappa$-neighborhood of $S^{\prime}$ in $Y$. Then $S:=f^{-1}\left(S_{0}\right)$ has size $\leq$ $\kappa d^{2 \kappa}\left|S^{\prime}\right|=O\left(\operatorname{cut}_{Y}\left(\left|A^{\prime}\right|\right)\right)=O\left(\operatorname{cut}_{Y}(|A|)\right)$, using (1.1). Denote by $C_{i}$ the preimage of $C_{i}^{\prime}$ by $f$.

We claim that $S$ is a separating set in $A$ between $C_{1} \backslash S, C_{2} \backslash S, \ldots, C_{m} \backslash S$. Suppose not: then there is a path $P$ in $A \backslash S$ between some $C_{i} \backslash S$ and $C_{j} \backslash S$ $(i \neq j)$. Then $f(P)$ is disjoint from $f(S)=S_{0}$, thus the $2 \kappa$-neighborhood $P^{\prime}$ of $f(P)$ in $A^{\prime}$ does not intersect $S^{\prime}$. Since $P^{\prime}$ is connected, this shows that some vertex of $f\left(C_{i} \backslash S\right) \subset C_{i}^{\prime}$ and some vertex of $f\left(C_{j} \backslash S_{0}\right) \subset C_{j}^{\prime}$ is connected by a path inside $A^{\prime} \backslash S^{\prime}$. This contradicts the fact that $C_{i}$ and $C_{j}$ are different components of $A^{\prime} \backslash S^{\prime}$.

We have seen that $|S|=O\left(\operatorname{cut}_{Y}(|A|)\right)$, and just seen that $S$ is a separating set in $A$ between $C_{1} \backslash S, C_{2} \backslash S, \ldots, C_{m} \backslash S$. On the other hand, $\left|C_{i} \backslash S\right| \leq\left|C_{i}\right| \leq$ $\kappa\left|C_{i}^{\prime}\right| \leq|A| / 2$.

We know and used three ways to rule out existence of regular maps between spaces: separation, Dirichlet harmonic functions, and growth. See [5] (where $\kappa$-regular maps are called $\kappa$-quasimonomorphisms). A fourth way is to use that asymptotic dimension is monotone under regular (and more generally, semi-regular) maps; see Section 6.

Separation and growth are monotone with respect to regular maps. Flows with finite energy can be pushed back and forth with a regular map. One should look for additional invariants. A partial motivation to study separation is to try to figure out the partial order given by regular maps on the collection of all spaces, or rather, on the familiar ones.

\section{Finite separation and regular maps}

A graph admits the finite separation property $(F S)$ iff $\operatorname{sep}_{G}(n)$ is a bounded function. Trees, and graphs that are roughly isometric to trees, are examples of graphs with finite separation. Yet these are not the only graphs with finite separation. Consider an infinite Sierpiński graph, which we define as some reasonable limit (say, local convergence, with root chosen to be always one of the three extremal vertices) of the sequence of finite Sierpiński graphs. This is an example of a graph with $\operatorname{sep}_{G}(n) \leq 3$ which is not roughly isometric to a tree, as it contains arbitrary large cycles. In this section we will try to understand the structure of graphs with finite separation. 
Theorem 2.1. If a bounded degree $G$ has finite separation then $G$ admits a regular map to the 3-regular tree.

Remark. It is easy to check that every locally finite graph has a regular map to a tree if the tree can have arbitrarily large degrees (because the uniform bound on the number of preimages in the definition of a regular map can be ignored by "blowing up" vertices to large enough stars).

The proof for Theorem 2.1 will proceed by showing that finite separation implies bounded treewidth.

Definition. Let $G$ be a finite graph, $T$ be a tree, and consider a family $\mathcal{V}=\left(V_{t}\right)_{t \in T}$ such that $V_{t} \subset V(G)$ for every $t$. We say that $(T, \mathcal{V})$ is a tree-decomposition of $G$ if the following hold:

(1) $\bigcup_{t \in V(T)} V_{t}=V(G)$;

(2) for every $e \in E(G)$ there is a $t$ such that both endpoints of $e$ are in $V_{t}$;

(3) for every $x \in V(G)$, the set $\left\{t \in V(T) \mid x \in V_{t}\right\}$ induces a connected subgraph of $T$.

The width of the tree decomposition is $\max _{t \in T}\left|V_{t}\right|-1$.

The treewidth of $G$, denoted by $\operatorname{tw}(G)$, is the minimum of the width of all tree decompositions of $G$.

An important property of tree decompositions is that for every edge $e=\{x, y\}$ of $T$, the set $V_{x} \cap V_{y}$ is a separating set between $\bigcup_{t \in C_{1}} V_{t} \backslash\left(V_{x} \cap V_{y}\right)$ and $\bigcup_{t \in C_{2}} V_{t} \backslash$ $\left(V_{x} \cap V_{y}\right)$, where $C_{1}$ and $C_{2}$ are the two components of $T \backslash e$.

The following theorem was proved by Robertson and Seymour [20]. See Theorem 12.4.4 in [11] for a proof and for more details on treewidth.

Theorem 2.2. For every $m$ there is a $k$ such that a graph of treewidth at least $k$ contains an m-by-m square grid as a minor.

Lemma 2.3. For any finite graph $G, \operatorname{cut}(G) \leq \operatorname{tw}(G)+1$. Conversely, for every $c$ there exists a $k$ such that if $\operatorname{tw}(G) \geq k$, then $\operatorname{cut}(G) \geq c$.

Proof. To see the first claim, let $(T, \mathcal{V})$ be a tree decomposition of $G$ of width $\operatorname{tw}(G)$. Choose $x \in V(T)$ so that the maximum of $\left|\bigcup_{t \in C} V_{t} \backslash V_{x}\right|$ over all components $C$ of $T \backslash x$ is minimal. It is easy to check that this maximum is at most $|V(G)| / 2$; on the other hand, this is the maximum size of components in $G \backslash V_{x}$. Hence $V_{x}$ separates, and the first part is proved.

For the other assertion, let $c$ be any positive integer. Choose $m \geq\left(4 c^{4}+1\right)^{1 / 2}\left(c^{2}+\right.$ 1) to be an integer, and $k$ as in Theorem 2.2 for this $m$. We will show that if $G$ has treewidth at least $k$, then it cannot be separated by $c$ vertices. 
By assumption and Theorem 2.2, such a $G$ contains an $m$ by $m$ grid $M$ as a minor. Let $G^{\prime}$ be a minimal subgraph of $G$ that can be contracted to a graph isomorphic to $M$. For each vertex $x$ of $M$, let $W_{x}$ be the set of vertices of $G^{\prime}$ that got contracted into $x$. Suppose that the vertices $x_{1}, x_{2}, \ldots, x_{m^{2}}$ of $M$ are ordered so that

$$
\left|W_{x_{1}}\right| \geq\left|W_{x_{2}}\right| \geq \cdots \geq\left|W_{x_{m^{2}}}\right| \text {. }
$$

Consider the set $X:=\left\{x_{1}, x_{2}, \ldots, x_{4 c^{4}}\right\}$ in $M$. Subdivide $M$ into (at least $4 c^{4}+1$ many) $c^{2}+1$ times $c^{2}+1$ pairwise disjoint subgrids (that is, subgraphs that are grids). At least one of these subgrids does not intersect $X$; let $M_{0}$ be a such subgrid. Let the vertices on the boundary of $M_{0}$ be $y_{1}, y_{2}, \ldots, y_{4 c^{2}}$. Our plan is to choose pairwise disjoint paths $P_{1}, \ldots, P_{2 c^{2}}$ from $X$ to $\left\{y_{1}, \ldots, y_{4 c^{2}}\right\}$ in $M$. Using these, for each $y_{i}$ that is the endpoint of some $P_{j}$, we will replace $W_{y_{i}}$ by a $\tilde{W}_{y_{i}}$ that is still connected, and has "many" vertices (and the same number for each such $y_{i}$ ). Then we will show that $\left(\bigcup \tilde{W}_{y_{i}}\right) \cup\left(\bigcup_{y \in M_{0}} W_{y}\right)$ is a subgraph of $G$ with no separation of size at most $c$. We will explain this construction in more detail later.

It is easy to check, using the isoperimetry of the square grid, that there is no separating set of size $\leq 2 c^{2}$ between $X$ and $\left\{y_{1}, \ldots, y_{4 c}\right\}$ in $M_{0}$. Hence, by the max-flow-min-cut theorem, $P_{1}, \ldots, P_{2 c^{2}}$ can be chosen. There is no restriction in assuming that their endpoints in $\left\{y_{1}, \ldots, y_{4 c^{2}}\right\}$ are respectively $y_{1}, \ldots, y_{2 c^{2}}$. For every $i \in\left\{1, \ldots, 2 c^{2}\right\}$, define $G_{i}$ to be the subgraph of $G^{\prime}$ induced by $\bigcup_{v \in P_{i}} V\left(W_{v}\right)$. The $G_{i}$ are connected (by the fact that $G_{i}$ can be contracted to $P_{i}$ ), and pairwise disjoint. For each $i \in\left\{1, \ldots, 2 c^{2}\right\}$, let $W_{y_{i}}^{\prime}$ be a connected subgraph of $G_{i}$ that contains $W_{y_{i}}$ and has size $\mu:=\min \left\{\left|W_{x_{1}}\right|, \ldots,\left|W_{x_{4 c^{4}}}\right|\right\}$. The choice of such $W_{y_{i}}^{\prime} \mathrm{s}$ is possible since $\left|W_{y_{i}}\right| \leq \mu$ and $\left|W_{x_{i}}\right| \geq \mu$ (note that $\left|W_{y_{i}}\right| \leq\left|W_{x_{j}}\right|$ for every $i, j \in\left\{1, \ldots, 4 c^{4}\right\}$, by the choice of $M_{0}$ ), and by the fact that the endpoint of each $P_{i}$ is some element of $\left\{x_{1}, \ldots, x_{4 c^{4}}\right\}$. For every $y \in M_{0} \backslash\left\{y_{1}, \ldots, y_{2 c^{2}}\right\}$, let $W_{y}^{\prime}:=W_{y}$. Finally, let $H$ be the subgraph of $G^{\prime}$ induced by $\bigcup_{y \in M_{0}} W_{y}^{\prime}$. We claim that $H$ is not separable by $\leq c$ vertices.

To see this, consider some separating set $S$ of size at most $c$ in $H$. Define $S_{M_{0}}$ as $\left\{y \in M_{0} \mid S \cap W_{y}^{\prime} \neq \emptyset\right\}$. Then $\left|S_{M_{0}}\right| \leq c$, and hence $M_{0} \backslash S_{M_{0}}$ has a component $C$ of size at least $\left|M_{0}\right|-c^{2}=\left(c^{2}+1\right)^{2}-c^{2}$. In particular, at least $c^{2}$ of the $y_{1}, \ldots, y_{2 c^{2}}$ are in $C$. The subgraph of $H$ induced by $\bigcup_{y \in C} W_{y}^{\prime}$ is clearly connected, and by the last sentence, it has at least $c^{2} \mu$ vertices. On the other hand, $\bigcup_{y \in M_{0} \backslash C} W_{y}^{\prime} \leq \mu\left|M_{0} \backslash C\right| \leq c^{2} \mu$. This shows that the complement of $\bigcup_{y \in C} W_{y}^{\prime}$ in $H \backslash S$ has size at least $c^{2} \mu$, while the union of all other components and $S$ has size at most $c^{2} \mu$. Thus $S$ does not cut $H$ to pieces of proportion $<1 / 2$. Since $S$ was arbitrary, this finishes the proof.

Proof of Theorem 2.1. The proof is based on the following lemma.

Lemma 2.4. Let $T$ be a bounded degree tree. Then there is a regular map $T \rightarrow \operatorname{reg} T_{2}$.

We leave the proof of the lemma as an (easy) exercise to the reader. 
The strong treewidth of a finite graph $H$, denoted by $\operatorname{stw}(H)$, is defined as follows. Take a so-called strong tree decomposition, which is defined to be a pair $(T, \mathcal{V})$, $\mathcal{V}=\left(V_{i}\right)_{i \in T}$, where $T$ is a tree, and $\left(V_{i}\right)_{i \in T}$ is a partition of $V(H)$, and for every edge $\{x, y\} \in E(H), x \in V_{i}, y \in V_{j}$, then either $i=j$ or $\{i, j\} \in E(T)$. The strong treewidth, $\operatorname{stw}(H)$, is defined as the minimum of $\max _{i \in T}\left|V_{i}\right|$ over all strong tree decompositions.

It is shown in [8] that for any family of bounded degree graphs, having bounded treewidth is equivalent to having bounded strong treewidth (see Theorem 95 in [7]). Take now the family $\Gamma$ of all finite connected subgraphs of $G$. From Lemma 2.3 we know that finite separation of $G$ implies that there exists a $c$ such that $\operatorname{tw}(H) \leq c$ for every $H \in \Gamma$. Hence there exists a $c^{\prime}$ such that $\operatorname{stw}(H) \leq c^{\prime}$ for every $H \in \Gamma$. Given an $H$, take a strong tree decomposition $(T, \mathcal{V})$. (We may assume that every $V_{i} \in \mathcal{V}$ is nonempty.) Then the map $\phi: H \rightarrow T, x \mapsto i$, where $x \in V_{i}$, is 1-regular. Observe that the maximal degree of $T$ is at most $c^{\prime} d$, otherwise there were adjacent vertices $s, t$ in $T$ such that there is no edge between $\bigcup_{i \in C_{1}} V_{i}$ and $\bigcup_{i \in C_{2}} V_{i}$, where $C_{1}$ and $C_{2}$ are the components one gets after deleting the edge $\{s, t\}$ from $T$. This would contradict the connectedness of $H$ (since every $V_{i}$ is nonempty). So, for every $H \in \Gamma$, there is a $\phi 1$-regular map into a tree of maximal degree at most $c^{\prime} d$. A standard compactness argument gives then that there is a 1-regular map from $G$ to a $c^{\prime} d$-regular tree. Since the composition of regular maps is regular, Lemma 2.4 finishes the proof.

\section{Products}

How does separation behave under products? Below is a lower bound that might be also the right upper bound. At least it is tight for the products $\mathbb{Z}^{d_{1}+d_{2}}=\mathbb{Z}^{d_{1}} \times \mathbb{Z}^{d_{2}}$.

Theorem 3.1. For $c=(7 / 8)^{1 / 2}$ and any finite graphs $G, H$,

$$
\operatorname{cut}(G \times H) \asymp \min \left(|H| \operatorname{cut}^{c}(G),|G| \operatorname{cut}^{c}(H)\right),
$$

where $\asymp$ means up to constant factors which are independent of $G$ and $H$.

Proof. Take a finite graph $G$, and let $K$ be a subset of $V(G)$. How can we tell if $K$ separates $G$ ? Here is how. Pick two vertices $v, u$ in $G$ at random independently and uniformly. Let $p$ be the probability that they are not in the same component of $G \backslash K$ (including the case when at least one of them is in $K$ ). Then $K$ separates only if $p>1 / 2$.

Now consider some set of vertices $K$ in $G \times H$. Let $\left(g_{1}, h_{1}\right)$ and $\left(g_{2}, h_{2}\right)$ be two randomly independently, uniformly chosen points in $G \times H$. Let $p_{1}$ be the probability that $\left(g_{1}, h_{1}\right)$ and $\left(g_{1}, h_{2}\right)$ are in different components of $g_{1} \times H-K$, let $p_{2}$ be the probability that $\left(g_{1}, h_{2}\right)$ and $\left(g_{2}, h_{2}\right)$ are in different components of $G \times h_{2}-K$, and let $p$ be the probability that $\left(g_{1}, h_{1}\right)$ and $\left(g_{2}, h_{2}\right)$ are in different 
components of $G \times H-K$. Clearly, $p \leq p_{1}+p_{2}$. If $K$ separates, then $p>1 / 2$, so at least one of $p_{1}, p_{2}$ is greater than $1 / 4$. Suppose that it is $p_{1}$. That implies that for at least $(1 / 8) \times|G|$ choices of $g_{1}$ in $G$ the probability that $h_{1}$ and $h_{2}$ are in different components of $g_{1} \times H-K \cap\left(g_{1} \times H\right)$ is at least $1 / 8$. So for those $g_{1}$ we have $K \cap\left(g_{1} \times H\right)$ separating $g_{1} \times H$ (for $c=(7 / 8)^{1 / 2}$, that is, the components after deletion have sizes at most $c$ times the original size). Hence, for such $g_{1}$ the cardinality of $K \cap\left(g_{1} \times H\right)$ is at least cut ${ }^{c}(H)$. Hence the cardinality of $K$ is at least $C|G| \operatorname{cut}^{c}(H)$ with some $C$. This proves the direction

$$
\operatorname{cut}(G \times H)>C \min \left(|H| \operatorname{cut}^{c}(G),|G| \operatorname{cut}^{c}(H)\right) .
$$

The other direction is obvious.

Recall (1.1). Using that, Theorem 3.1 provides us with a lower bound for the separation function of product graphs. However, it does not settle the question regarding the separation of a product of infinite graphs, because it does not provide us with an upper bound for the separation of a finite graph $F \subset G \times H$ that is not a product.

Theorem 3.2. Let $G_{1}$ and $G_{2}$ be two (possibly infinite) graphs. Then

$$
\operatorname{sep}_{G \times H}(N)=\Omega\left(\max _{k} \min \left(N / k \operatorname{sep}_{G}(k), k \operatorname{sep}_{H}(N / k)\right)\right) .
$$

In particular,

$$
\operatorname{sep}_{G \times \mathbb{Z}}\left(m^{2} / \operatorname{sep}_{G}(m)\right)=\Omega(m)
$$

Proof. The first half is straightforward from Theorem 3.1 (using also (1.1)). For the second assertion, let $N=m^{2} / \operatorname{sep}_{G}(m)$. The numbers whose minimum we take on the right side are equal (up to constant factor) if $k=m / \operatorname{sep}_{G}(m)$, hence the maximum on the right, given by this case, is $m$.

Corollary 3.3. We have

$$
\begin{aligned}
\operatorname{sep}_{\mathbb{Z}^{d}}(n) & \asymp n^{(d-1) / d}, \\
\operatorname{sep}_{\mathbb{H}^{2} \times \mathbb{H}^{2}}(n) & =\Omega\left(n^{1 / 2} / \log n\right), \\
\operatorname{sep}_{\mathbb{R} \times \mathbb{H}^{2}} & =\Omega(n \log n)^{1 / 2} .
\end{aligned}
$$

Proof. The lower bound for $\operatorname{sep}_{\mathbb{Z}^{d}}(n)$ follows from the finite separation of $\mathbb{Z}$ using induction and Theorem 3.2. The upper bound is given in Proposition 4.1 (see the details there), and by a similar argument one can get the upper bound for $\mathbb{R} \times \mathbb{H}^{2}$ (also using Theorem 3.5).

For $\mathbb{H}^{2} \times \mathbb{H}^{2}$, we use that the separation of $\mathbb{H}^{2}$ is $\log n$ by Theorem 3.2. By symmetry we have $\max _{k} \min \left(n / k \operatorname{sep}_{\mathbb{H}^{2}}(k), k \operatorname{sep}_{\mathbb{H}^{2}}(n / k)\right)=n^{1 / 2} \log n^{1 / 2} \asymp n^{1 / 2} \log n$.

Finally, the lower bound for $\operatorname{sep}_{\mathbb{R} \times \mathbb{H}^{2}}$ is straightforward from the $G \times \mathbb{Z}$ part of Theorem 3.2. 
The previous lower bound on $\operatorname{sep}_{\mathbb{H}^{2} \times \mathbb{H}^{2}}$ will be improved in Theorem 5.1.

Conjecture 3.4. $\operatorname{sep}_{G \times \mathbb{Z}}(n)$ is always equal to the lower bound in Theorem 3.2.

This means that up to constants $\operatorname{sep}_{G \times \mathbb{Z}}\left(m^{2} / \operatorname{sep}_{G}(m)\right) \asymp m$.

The value of $\operatorname{sep}_{\mathbb{H}^{2} \times \mathbb{R}}(n)$ is a particular case of that, the lower bound was given in Corollary 3.3.

We are bugged by not being able to settle the question of the separation of a graph of the form $G \times \mathbb{Z}$, where the separation function of $G$ is known. If the worst case is a product subgraph of $G \times \mathbb{Z}$ then $\operatorname{sep}_{G \times \mathbb{Z}}\left(n^{2} / \operatorname{sep}_{G}(n)\right) \asymp n$ would be the answer. We can prove this for the case when $G$ has finite separation. To obtain $\operatorname{sep}_{G \times \mathbb{Z}}(n) \asymp \sqrt{n}$, take a regular map from $G$ to a regular tree $T$ (using Theorem 2.1). This defines a regular map from $\mathbb{Z} \times G$ to $\mathbb{Z} \times T$. The separation of the latter is $\sqrt{n}$; see Lemma 7.2 in [5], hence we have the $\sqrt{n}$ upper bound. For the lower bound, apply Theorem 3.1.

Denote by $T$ the binary tree, we have:

\section{Theorem 3.5.}

$$
\operatorname{sep}_{T \times T}(n) \asymp \frac{n}{\log n} .
$$

Remark. This implies an upper bound of $n / \log n$ for the product of any two bounded valence trees (using Theorem 2.4), and a lower bound for $\mathbb{H}^{2} \times \mathbb{H}^{2}$.

The proof is similar to that of Lemma 7.2 in [5].

Proof. Lower bound first. A useful representation of $T \times T$ is as follows. Consider sequences that are finite, consist of 0's and 1's, and have a decimal point. Two of these are neighbors if one is obtained from the other by adding a digit either on the right or on the left. We will use this model for $T \times T$. Let $B_{k}$ be the set of points in $T \times T$ that are at distance $k$ from the root (which is ". "). These are just the length $\leq k$ binary sequences with a dot at some place inside. Suppose that $W$ is a separating set for $B_{k}$. Pick two length $2 k$ binary sequences at random, with the dot in the center of each. Let these be called $a$ and $b$. At random, pick a $k$-length subword of $a$, and denote it by $a^{\prime}$, and pick a $k$-length subword of $b$, denoted by $b^{\prime}$. Then $a^{\prime}$ and $b^{\prime}$ are just independent uniformly selected random vertices in $B_{k} \backslash B_{k-1}$. Let $a_{1}$ be the left half of $a$, that is, the part of $a$ to the left of the dot. This is a point in $B_{k}$. Let $a_{2}$ be the right half of $a$. Same for $b=b_{1} \cdot b_{2}$, all have length $k$. Consider the following path from $a^{\prime}$ to $b^{\prime}$ through $a_{2}$ and $b_{1}$. Delete the leftmost digit in $a^{\prime}$. Then add to the right the next digit of $a_{2}$. Do so repeatedly until you reach $a_{2}$. Then delete the rightmost digit, and add on the left the digit of $b_{1}$. Continue that until you reach $b_{1}$. Then delete digits on the left and add on the right the digits of $b_{2}$ until you reach $b^{\prime}$. In this path you see length $k$ and $k-1$ subwords of the words $a, b_{1} \cdot a_{2}$, and $b$. With probability at least $1 / 4$, say, you must pass through the separating set $W$ in this process. Since $a$, $b_{1} \cdot a_{2}$ and $b$ are equally distributed, the probability that $b$ contains a subword in $W$ (of length $k$ or $k-1$ ) is at least $1 / 12$. There are $4^{k}$ different possible $b$ 's. Each word 
in $W$ with length $k$ or $k-1$ is contained in $2^{k}$ or $2 \times 2^{k}$ different $b$ 's. Hence the size of $W$ is at least $2^{k} / 24$. But $\left|B_{k}\right| \asymp k \times 2^{k}$. So $|W|>C\left|B_{k}\right| / \log \left(\left|B_{k}\right|\right)$.

Now the upper bound. Let $S$ be a finite set of points in $T \times T$. We shall find a separating set for $S$ of size $C n / \log n$, where $n=|S|$. Let $p_{1}$ and $p_{2}$ be the projections into the factors. Let $D\left(p_{1}(v)\right), D\left(p_{2}(v)\right)$ be the distance from the root in the respective factors. Let $m_{1}$ be the median of $D\left(p_{1}(v)\right)$ on $S$, and let $m_{2}$ be the median of $D\left(p_{2}(v)\right)$ on $S$. Let $A_{1}(m)$ be the set of vertices in $S$ such that $D\left(p_{1}(v)\right)=$ $m_{1}$, and similarly for $A_{2}(m)$. If the size of $A_{1}\left(m_{1}\right)$ is less than $100 n / \log n$, then we can take $A_{1}\left(m_{1}\right)$ as the separating set. Otherwise, let $k_{1}^{+}$be the least $k \geq m_{1}$ such that $\left|A_{1}\left(k_{1}^{+}\right)\right|<100 n / \log n$, let $k_{1}^{-}$be the greatest $k \leq m_{1}$ such that $\left|A_{1}\left(k_{1}^{-}\right)\right|<$ $100 n / \log n$. Similarly, define $k_{2}^{-}$and $k_{2}^{+}$. The separating set is $A=A_{1}\left(k_{1}^{-}\right) \cup$ $A_{1}\left(k_{1}^{+}\right) \cup A_{2}\left(k_{2}^{-}\right) \cup A_{2}\left(k_{2}^{+}\right)$. The size of $A$ is obviously less than $400 n / \log n$. We just need to check that $A$ separates well. Let $K$ be a component of $S \backslash A$, and let $v$ be some vertex in $K$. If $D\left(p_{1}(v)\right)<k_{1}^{-}$, then every vertex $w$ in $K$ satisfies this inequality. Because $m_{1} \geq k_{1}^{-}$is the median for $D\left(p_{1}(z)\right)$, this clearly implies that $|K| \leq n / 2$. So we may assume that $D\left(p_{1}(v)\right) \geq k_{1}^{-}$holds for every vertex $v$ in $K$. Similarly, one gets $D\left(p_{1}(v)\right) \leq k_{1}^{+}, k_{2}^{-} \leq D\left(p_{2}(v)\right) \leq k_{2}^{+}$. A connected component of $t \in T: k^{-} \leq D(t) \leq k^{+}$has cardinality exactly $2^{k^{+}-k^{-}-1}$. Hence it follows that $|K| \leq 2^{k_{1}^{+}-k_{1}^{-}} \times 2^{k_{2}^{+}-k_{2}^{-}}$. But note that $k_{j}^{+}-k_{j}^{-} \leq \log (n) / 100+1$ for $j=1,2$, because for each $k$ in the range $k_{j}^{-}<k<k_{j}^{+}$we have $\left|A_{j}(k)\right| \geq 100 n / \log n$. This gives $|K| \leq 4 \times 2^{\log n / 50}<n / 2$. Hence $A$ is a separating set.

The proof shows also that $\operatorname{sep}_{T \times T \times T}(n) \asymp n / \log n$, and the same for any $T \times$ $T \times \cdots \times T$.

\section{Hyperbolic graphs}

Before proceeding to the study of more general hyperbolic graphs, we determine the separation function of the hyperbolic space $\mathbb{H}^{d}$. This follows from the method in [17], [18], which can also be used to give a proof for the separation of $\mathbb{R}^{d}$.

Proposition 4.1. For $d=2$, $\operatorname{sep}_{\mathbb{H}^{d}} \asymp \log n$, and for $d \geq 3$, $\operatorname{sep}_{\mathbb{H}^{d}} \asymp n^{(d-2) /(d-1)}$. For $d \geq 1, \operatorname{sep}_{\mathbb{Z}^{d}} \asymp n^{(d-1) / d}$.

Proof. Suppose that $d \geq 3$. The lower bound follows from the fact that $\mathbb{R}^{d-1}$ embeds isometrically into $\mathbb{H}^{d}$.

For the upper bound, we adapt the proof of [17] to our setting.

Take a connected fundamental domain $Q$ of $\mathbb{H}^{d}$ by some group of isometries acting co-compactly and properly discontinuously on $\mathbb{H}^{d}$. The translates of $Q$ by this group give a tiling $\mathcal{T}$ of $\mathbb{H}^{d}$; denote the corresponding dual graph by $G$. For a vertex $x \in G$, let $\tau(x)$ be the corresponding tile. Clearly $G$ is transitive and roughly 
isometric to $\mathbb{H}^{d}$. We want to show that $G$ has separation $n^{(d-2) /(d-1)}$ if $d \geq 3$, and $\log n$ if $d=2$.

So let $H$ be a finite subgraph of $G$, and $\left.\mathcal{T}\right|_{H}$ be the union of the corresponding set of tiles in $\mathbb{H}^{d}$. Denote by $o$ a centerpoint of $\left.\mathcal{T}\right|_{H}$, that is, a point with the property that any hyperplane $P$ through $o$ cuts $\left.\mathcal{T}\right|_{H}$ into two pieces whose volumes are the same up to a factor $d$. (See [17] for references showing the existence of centerpoints.) In particular, $\{x \in H \mid \tau(x)$ intersects $P\}=: S$ separates $H$. So we want to bound $|S|$.

Choose $P$ randomly and uniformly of all hyperspaces through $o$. Look at $\mathcal{T}$ in the corresponding Poincaré ball model; we may assume that $o$ is the origin (and then $S$ is the intersection of a Euclidean hyperspace with the open unit ball). Let the tile containing the origin be $t$. If $d \geq 3$, the expected number of tiles in $\left.\mathcal{T}\right|_{H}$ intersected by $P$ can be estimated as follows. The set of all tiles in $\mathcal{T}$ at graphdistance $k$ from $t$ is of order $c^{(d-1) k}$ with some $c>1$; the set of those intersecting $P$ is of order $c^{(d-2) k}$ (here we are using the fact that the tiles all have the same volume, hence the same $c$ works for both cases). Hence, if $m_{k}$ is the number of tiles in $\left.\mathcal{T}\right|_{H}$ intersecting $P$ and at graph distance $k$ from $t$, we obtain $\mathbf{E}(|S|)=$ $O\left(\sum_{k=1}^{\infty} m_{k} c^{(d-2) k} / c^{(d-1) k}\right)=O\left(\sum m_{k} c^{-k}\right)$. This is maximized when $H$ is a ball, in which case we have $\mathbf{E}(|S|)=O\left(c^{(d-1) \log n} c^{-\log n}\right.$ ) (where the base of the logarithm is $c^{d-1}$ ). Hence, there exists a $P$ such that the corresponding $S$ has $O\left(c^{(d-2) \log n}\right)=O\left(n^{(d-2) /(d-1)}\right)$ elements, which is what we wanted to prove.

The proofs for $\mathbb{H}^{2}$ and for $\mathbb{Z}^{d}$ proceed similarly, so we omit the details.

From this proof technique of [17], one also gets that a graph which can be sphere packed in $\mathbb{R}^{d}$ such that the spheres in the packing have bounded ratios, have separation at most $O\left(n^{(d-1) / d}\right)$. This implies, e.g., that $\mathbb{Z}^{d+1}$ cannot be sphere packed in $\mathbb{R}^{d}$. For more on this, see [6].

Remark. In [15] the following is proved. Say that a graph $G$ has growth rate $d$ if every ball of radius $r$ in $G$ contains at most $r^{d}$ vertices. Then there is an injective graph homomorphism from $G$ to $\mathbb{Z}_{\infty}^{d \log d}$, where $\mathbb{Z}_{\infty}^{d \log d}$ is the graph on $\mathbb{Z}^{d \log d}$ where two vertices are adjacent if each of their coordinates differ by at most one. Together with the method of the previous proof, we can conclude that $\operatorname{sep}_{G}(n)=$ $O\left(n^{(d \log d-1) /(d \log d)}\right)$ for such a $G$.

In the standard hyperbolic plane $\mathbb{H}^{2}$, triangles do not have the same properties as in the Euclidean plane. For instance, in the Euclidean plane, in any large isosceles triangle, the midpoint of the hypotenuse is far away from the other two sides. This cannot happen in hyperbolic space. That observation led E. Rips to the following definition.

Definition. Let $G=(V, E)$ be a graph. Given three vertices $u, v, w \in V$, pick geodesics between any two to get a geodesic triangle. Denote the geodesics by 
$[u, v],[v, w],[w, u]$. Say the triangle is $\delta$-thin if for any $v^{\prime} \in[u, v]$,

$$
\min \left(d\left(v^{\prime},[w, u]\right), d\left(v^{\prime},[v, w]\right)\right) \leq \delta .
$$

$G$ is said to be $\delta$-hyperbolic if there is some $\delta \geq 0$ such that all geodesic triangles in $G$ are $\delta$-thin.

Hyperbolic groups were introduced by Gromov [13]. They are among the central objects in geometric group theory.

We do not have any general upper bounds on the separation of hyperbolic graphs. Still, we have the following gap theorem.

Theorem 4.2. Let $G=(V, E)$ be a graph which is $\Delta$-hyperbolic and has maximal degree $M<\infty$. Let $N$ be some integer. There is a $c>0$, which depends only on $M, N$ and $\Delta$, such that if

$$
\operatorname{sep}_{G}(n)<c \log n \text { for all } n>N,
$$

then $G$ is roughly-isometric to a tree.

Lemma 4.3. Let $G=(V, E)$ be a graph which is $\Delta$-hyperbolic and has maximal degree $M<\infty$, and let $v_{0}$ be some vertex of $G$.

Let $u$ s write $u \sim v$ whenever $u$, $v$ are two vertices of $G$ with $d\left(u, v_{0}\right)=d\left(v, v_{0}\right)$ and there is a sequence of vertices $u=u_{1}, u_{2}, \ldots, u_{k}=v$ in $G$ with $d\left(u_{j}, v_{0}\right)=$ $d\left(u, v_{0}\right)$ and $d\left(u_{j}, u_{j+1}\right) \leq 2 \Delta$ for each $j=1, \ldots, k-1$.

Let $N$ be some integer. There are $D, c>0$, which depend only on $M, N$ and $\Delta$ such that if (4.1) holds, then $d(u, v)<D$ whenever $u \sim v$.

Proof. For each vertex $v \neq v_{0}$, let $\sigma_{1} v$ be one of the neighbors of $v$ that is closer to $v_{0}$, and set $\sigma_{1} v_{0}=v_{0}$. Inductively, define $\sigma_{n+1} v=\sigma_{1} \sigma_{n} v$, and set $\sigma(v)=$ $\left\{v, \sigma_{1} v, \sigma_{2} v, \ldots, v_{0}\right\}$. Note that this is a geodesic from $v$ to $v_{0}$, and $\sigma(u) \subset \sigma(v)$ holds whenever $u \in \sigma(v)$.

Now assume that $u_{1}, u_{2}, \ldots, u_{k}$ is a sequence of vertices such that $d\left(u_{j}, v_{0}\right)=$ $d\left(u_{1}, v_{0}\right)$ and $d\left(u_{j}, u_{j+1}\right) \leq 2 \Delta$ for each $j=1, \ldots, k-1$. Also assume that (4.1) holds for some $c$ satisfying

$$
c^{-1}>(2 \Delta+3) \log M
$$

and for some $N$. We need to show that $d\left(u_{1}, u_{k}\right)<D$ for some $D=D(\Delta, M, N)$.

It follows from (4.2) that

$$
c \log 11+c \log Q+c Q \log M<\frac{Q-\Delta}{2 \Delta+3}
$$

holds for all sufficiently large $Q$. So let $Q$ be an integer large enough to satisfy (4.3) and

$$
10 M^{Q}>\max \{N, 2 \Delta\} .
$$


Also set

$$
L=10 M^{Q}, \quad R=d\left(u_{1}, v_{0}\right) .
$$

It is clear that we may assume $R>L+Q$, for otherwise $D=2(L+Q)$ suffices. Let $t$ be the largest integer in $\{1, \ldots, k\}$ such that the cardinality of $A=\left\{\sigma_{L+Q} u_{j} \mid\right.$ $j=1, \ldots, t\}$ is at most $Q$. See figure below. Our plan is to prove that $|A|<Q$. The definition of $t$ then shows that $t=k$. As we shall see, the diameter of $A$ is bounded by $2 \Delta|A|$. Hence it follows from $t=k$ and $|A|<Q$ that $d\left(u_{1}, u_{k}\right) \leq D$ with $D=2 \Delta Q+2 L+2 Q$ (consider the path from $u_{1}$ to $u_{k}$ constructed by following $\sigma\left(u_{1}\right)$ to $A$, taking the shortest path to $A \cap \sigma\left(u_{k}\right)$, and then following $\sigma\left(u_{k}\right)$ to $\left.u_{k}\right)$, and the lemma follows. So the main task is to show that $|A|<Q$.

For every $w \in A$ let $x_{w}$ be one of the $u_{j}$ 's in $\left\{u_{1}, \ldots, u_{t}\right\}$ so that $w=\sigma_{L+Q} x_{w}$, and let $s_{w}$ be the segment of $\sigma\left(x_{w}\right)$ between $x_{w}$ and $w$; that is, $s_{w}=\left\{\sigma_{j} x_{w} \mid j=\right.$ $1,2, \ldots, L+Q\}$.

Let $U$ be the set of vertices of $G$ that are at distance at most $Q$ from $A$ or that are in one of the segments $s_{w}, w \in A$, and let $H$ be the graph obtained by restricting $G$ to $U$. By construction, the segments $s_{w}, w \in A$ are disjoint, and consequently,

$$
|U| \geq|A|(L+Q)>|A| L=10|A| M^{Q} .
$$

On the other hand, a ball of radius $Q$ in $G$ has clearly no more than $M^{Q}$ vertices, and it follows that

$$
|U| \leq|A|\left(L+M^{Q}\right)=11|A| M^{Q} .
$$

From (4.1) and $10 M^{Q}>N$ it follows that there is a set of vertices $U_{0} \subset U$ with

$$
\left|U_{0}\right| \leq c \log |U| \leq c \log \left(11|A| M^{Q}\right)
$$

such that every component of $H-U_{0}$ has less than $6|A| M^{Q}$ vertices.

We are trying to prove that $|A|<Q$. By construction $|A| \leq Q$, so assume that $|A|=Q$. Therefore, from (4.4) and (4.3) it follows that

$$
\left|U_{0}\right| \leq c \log 11+c \log Q+c Q \log M<\frac{Q-\Delta}{2 \Delta+3} \leq Q / 3 .
$$

Let $A^{\prime}$ be the set of $w \in A$ such that the segment $s_{w}$ does not meet $U_{0}$. Because these segments are disjoint, and $|A|=Q$, it follows from (4.5) that

$$
\left|A^{\prime}\right|>2 Q / 3 \text {. }
$$

Let $r$ be an integer in the range $[R-L-Q, R-L-\Delta]$, and let

$$
V_{r}=\left\{v \in V|| d\left(v, v_{0}\right)-r \mid \leq \Delta\right\} .
$$

We claim that there is a path whose vertices are in $V_{r} \cap U$ that intersects each $s_{w}$, $w \in A$. For each $j=1, \ldots, t-1$, let $y_{j}$ be the vertex on $\sigma\left(u_{j}\right)$ that has distance $r$ from $v_{0}$. Consider the triangle in $G$ formed by taking $\sigma\left(u_{j}\right), \sigma\left(u_{j+1}\right)$ and a shortest 
curve $\alpha_{j}$ joining $u_{j}$ and $u_{j+1}$. Since $G$ is $\Delta$-hyperbolic, there is a vertex $z_{j+1}$ in $\alpha_{j} \cup \sigma\left(u_{j+1}\right)$ whose distance to $y_{j}$ is at most $\Delta$. Because the length of $\alpha_{j}$ is at most $2 \Delta$, the distance from $\alpha_{j}$ to $v_{0}$ is at least $R-\Delta$. On the other hand,

$$
d\left(z_{j+1}, v_{0}\right) \leq d\left(z_{j+1}, y_{j}\right)+d\left(y_{j}, v_{0}\right) \leq \Delta+r<R-\Delta \text {, }
$$

and it follows that $z_{j+1} \notin \alpha_{j}$. Hence $z_{j+1} \in \sigma\left(u_{j+1}\right)$, and there is a path $\beta_{j}$ in $U$ from $y_{j}$ to $z_{j+1}$ with all vertices in $V_{r}$ (since $d\left(z_{j+1}, v_{0}\right)<R-L$ ). Let $\gamma_{j+1}$ be the arc of $\sigma\left(u_{j+1}\right)$ connecting $z_{j+1}$ with $y_{j+1}$. It is now clear that the union of all the $\operatorname{arcs} \beta_{j}$ and $\gamma_{j}$ is a curve whose vertices are in $V_{r} \cap U$ that intersects each $s_{w}, w \in A$. This construction with $r=R-Q-L$ also shows that the diameter of $A$ is bounded by $2 \Delta|A|$, as we have promised.

Let $m$ be the largest integer smaller than $(Q-\Delta) /(2 \Delta+2)$. Set $r_{j}=R-Q-$ $L+2 j(\Delta+1)$ for $j=0,1, \ldots, m$, and note that the sets $V_{r_{j}}$ are disjoint. Since

$$
\left|U_{0}\right|<m+1
$$

follows from (4.5), there must be some such $r_{j}$ so that $U_{0}$ does not meet $V_{r_{j}}$. From the above it then follows that there is a connected component of $H-U_{0}$ that intersects each $s_{w}, w \in A$. Consequently, there is a connected component $X$ of $H-U_{0}$ that contains each $s_{w}, w \in A^{\prime}$. The number of vertices of $X$ is at least

$$
\left|A^{\prime}\right| L \geq 2 Q L / 3=20|A| M^{Q} / 3>6|A| M^{Q} .
$$

This contradicts the definition of $U_{0}$, and the contradiction establishes $|A|<Q$ and the lemma.

Lemma 4.4. Let $G$ be a $\Delta$-hyperbolic graph, let $v_{0}$ be some vertex in $G$, and let be the equivalence relation defined in Lemma 4.3. Let $V_{1}$ be the set of vertices of $G$ whose distance from $v_{0}$ is divisible by $3 \Delta+1$, and let $\tilde{V}=V_{1} / \sim$ be the set of equivalence classes of $\sim$ in $V_{1}$. Let $\widetilde{G}=(\tilde{V}, \widetilde{E})$ be defined by letting $[\tilde{v}, \tilde{u}] \in \widetilde{E}$ whenever for some $v \in \tilde{v}, u \in \tilde{u}$ there is a geodesic passing through $v_{0}, v, u$ and $d(v, u)=3 \Delta+1$. Then $\widetilde{G}$ is a tree.

Proof. Let $\tilde{d}$ denote the distance in $\widetilde{G}$, and set $\tilde{v}_{0}=\left\{v_{0}\right\} \in \tilde{V}$, the equivalence class of $v_{0}$. It is clear that if $v \in \tilde{v} \in \tilde{V}$, then $\tilde{d}\left(\tilde{v}, \tilde{v}_{0}\right)=(3 \Delta+1) d\left(v, v_{0}\right)$.

Let $\tilde{v} \in \tilde{V}-\left\{\tilde{v}_{0}\right\}$. By construction, $\tilde{v}$ has no neighbor $\tilde{u}$ with $\tilde{d}\left(\tilde{v}, \tilde{v}_{0}\right)=\tilde{d}\left(\tilde{u}, \tilde{v}_{0}\right)$. We claim that there is exactly one neighbor $\tilde{u}$ of $\tilde{v}$ such that $\tilde{d}\left(\tilde{v}, \tilde{v}_{0}\right)=\tilde{d}\left(\tilde{u}, \tilde{v}_{0}\right)+1$. Indeed, let $v_{1}, v_{2} \in \tilde{v}$, and suppose that $d\left(v_{1}, v_{2}\right) \leq 2 \Delta$. Let $s_{1}$ be a shortest path from $v_{1}$ to $v_{0}$, and let $s_{2}$ be a shortest path from $v_{2}$ to $v_{0}$. Let $u_{1}$ be the vertex on $s_{1}$ whose distance from $v_{1}$ is equal to $3 \Delta+1$, and let $u_{2}$ be the vertex on $s_{2}$ whose distance from $v_{2}$ is $3 \Delta+1$. Let $s_{3}$ be a shortest segment joining $v_{1}$ and $v_{2}$. Because $G$ is $\Delta$-hyperbolic, there is a vertex $w$ in $s_{3} \cup s_{2}$ whose distance to $u_{1}$ is at most $\Delta$. But the length of $s_{3}$ is at most $2 \Delta$, and hence the distance from $s_{3}$ to $u_{1}$ is at least $\Delta+1$. Thus, 
$w \notin s_{3}$ and $w \in s_{2}$. Since $\left|d\left(w, v_{0}\right)-d\left(u_{2}, v_{0}\right)\right|=\left|d\left(w, v_{0}\right)-d\left(u_{1}, v_{0}\right)\right| \leq \Delta$, and $w \in s_{2}$, we get $d\left(w, u_{2}\right) \leq \Delta$. So $d\left(u_{1}, u_{2}\right) \leq d\left(u_{1}, w\right)+d\left(w, u_{2}\right) \leq 2 \Delta$, which implies that $u_{1}$ and $u_{2}$ belong to the same equivalence class in $\widetilde{V}$. It now follows that $\tilde{v}$ has exactly one neighbor $\tilde{u} \in \tilde{V}$ with $\tilde{d}\left(\tilde{v}, \tilde{v}_{0}\right)=\tilde{d}\left(\tilde{u}, \tilde{v}_{0}\right)+1$.

Suppose that $\widetilde{G}$ is not a tree. Then there is a simple closed loop $\widetilde{C}$ in $\widetilde{G}$. Consider a vertex $\tilde{v}$ in $\widetilde{C}$, where $\tilde{d}\left(\tilde{v}, \tilde{v}_{0}\right)$ is maximal. The two edges adjacent to $\tilde{v}$ in $\widetilde{C}$ must connect $\tilde{v}$ with neighbors $\tilde{u}$ with $\tilde{d}\left(\tilde{v}, \tilde{v}_{0}\right) \gtreqless \tilde{d}\left(\tilde{u}, \tilde{v}_{0}\right)$, but $\tilde{v}$ has at most one such neighbor. This contradicts the existence of $\overline{\widetilde{C}}$, and hence $\widetilde{G}$ is a tree.

Proof of Theorem 4.2. Let $v_{0}$ be some vertex in $G$, and let $V_{1}, \widetilde{G}$ be as in Lemma 4.4. For each $v \in V$ choose some geodesic $\sigma(v)$ from $v$ to $v_{0}$, and let $v^{\prime}$ be the vertex closest to $v$ in $\sigma(v) \cap V_{1}$. Let $\pi v$ denote the equivalence class of $v^{\prime}$ in $\widetilde{V}=V / \sim$. It is an easy exercise to check that the $\Delta$-hyperbolicity of $G$ implies that $\pi$ is a contraction. On the other hand, it follows from Lemma 4.3 that $\tilde{d}(\pi v, \pi u) \geq C^{-1} d(v, u)-C$ holds for some $C>0$ and every $v, u \in V$. We conclude that $\pi$ is a rough-isometry. Now Lemma 4.4 shows that $\widetilde{G}$ is a tree, which completes the proof of the theorem.

Question 4.5. Show that for any planar hyperbolic graph $G$,

$$
\operatorname{sep}_{G}(n)<C \log n
$$

for some $C(G)=C>0$.

Note that, by Proposition 4.1, this cannot be true in general if we omit planarity.

\section{More on regular maps}

Let $T$ denote the binary tree. The next result is shown to be true in [9], even with a quasi-isometric embedding.

Theorem 5.1. $\mathbb{H}^{2} \rightarrow_{\mathrm{reg}} T \times T$.

Using Theorem 3.5 and the remark after its proof, the previous theorem implies that $\operatorname{sep}_{\mathbb{H}^{2} \times \mathbb{H}^{2}}(n) \asymp n / \log n$ because $T \times T \rightarrow_{\text {reg }} \mathbb{H}^{2} \times \mathbb{H}^{2} \rightarrow_{\text {reg }} T \times T \times T \times T$.

Question 5.2. Is $\mathbb{H}^{2} \rightarrow_{\text {reg }} T \times \mathbb{Z}$ ?

There may be something special about the $n / \log (n)$ separation function. The following problems are weaker than Question 1.1.

Question 5.3. (1) Suppose that $G, H$ have separation functions $\leq n / \log n$. Is it true that $G \times H$ has separation function $\leq n / \log n$ ?

(2) Is it true that balls in infinite transitive graphs have

$$
\operatorname{cut}(B(r)) \leq|B(r)| / \log |B(r)| ?
$$


The last question, if true, would answer negatively an old question of the first author, namely, if there is any Cayley graph where the balls form an expander family (see [4]). Without assuming transitivity, the answer to this question is clearly no: take some infinite tree with expanders put on each set of points at the same distance from the origin (as in [4]).

We have found that $\operatorname{sep}_{\mathbb{H}^{2} \times \mathbb{R}}(n)$ is at least $\sqrt{n \log n}$ (Corollary 3.3). This implies that $\mathbb{H}^{2} \times \mathbb{R}$ does not have a regular map to $\mathbb{H}^{3}$ which has separation only of order $\sqrt{n}$ (Proposition 4.1).

Question 5.4. Is $\mathbb{H}^{3} \rightarrow_{\text {reg }} \mathbb{H}^{2} \times \mathbb{R}$ ?

We doubt that the answer would be positive, but as we mention in Section 1.1, we know only four ways to rule out regular maps: separation, Dirichlet harmonic functions, asymptotic dimension and growth. Neither does the job here.

\section{Semi-regular maps}

In a private communication, Yehuda Shalom asked: Is it true that a Cayley graph of an amenable group which is not virtually cyclic admits a bilipschitz embedding of $\mathbb{Z}^{2}$ ? Yehuda suggested that the lamplighter group $(\mathbb{Z} / 2 \mathbb{Z})_{2} \prec \mathbb{Z}$ is a counterexample (see e.g. [19] for a detailed definition).

Here is a proof not using any of the arguments applied in the sections before but rather using the notion of semi-regular maps defined below. This will allow us to prove that there is no regular map $\mathbb{Z}^{2} \rightarrow_{\text {reg }} \operatorname{Lamplighter}(\mathbb{Z})$.

Definition. Call a map $f: X \rightarrow Y$ semi-regular, denoted by $X \rightarrow_{\text {s-reg }} Y$, if $f$ is Lipschitz and for every $r$ there is a $c(r)<\infty$ such that for every $y \in Y$ every connected component of $f^{-1}(B(y, r))$ has diameter at most $c(r)$.

It is an easy exercise to verify that a composition of semi-regular maps is semiregular.

Also, a regular map is semi-regular (we assume bounded degree; there are analogous definitions appropriate for general metric spaces).

Note that the canonical map Lamplighter $(\mathbb{Z}) \rightarrow \mathbb{Z}$ (location of the lamplighter) is semi-regular, with $c(r)$ of order $r 2^{r}$.

Proposition 6.1. There is no semi-regular map $\mathbb{Z}^{2} \rightarrow_{\text {s-reg }} \mathbb{Z}$.

By combining with the above, it follows that there is no semi-regular map $\mathbb{Z}^{2} \rightarrow_{\text {s-reg }}$ Lamplighter $(\mathbb{Z})$. In particular, there is no regular map $\mathbb{Z}^{2} \rightarrow_{\text {reg }}$ Lamplighter $(\mathbb{Z})$. 
Proof. Let $g: \mathbb{Z}^{2} \rightarrow \mathbb{Z}$ be Lipschitz. We look at "quasi-level sets" of $g$, and show that there is some quasi-connected component of a quasi-level set that is large (at this point, this is a vague statement; the precise formulation will become clear shortly). Take $k$ large, and consider the map $a(z):=\lfloor(z / k)\rfloor, a: \mathbb{Z}->\mathbb{Z}$. Fix $k$ large enough so that $\left|a(g(z))-a\left(g\left(z^{\prime}\right)\right)\right|<2$ if $z$ and $z^{\prime}$ are within distance 2 in $\mathbb{Z}^{2}$. Let $X$ be the graph obtained from $\mathbb{Z}^{2}$ by adding diagonals. Given $r \in \mathbb{Z}$, let $S(r)$ denote the set of connected components in $X$ of $\{x \in X \mid a(g(z))=r\}$. Let $S *:=\bigcup_{r \in \mathbb{Z}} S(r)$. This is a partition of $X$. Note that if $S \in S(r)$ is finite, then there is a unique $S^{\prime} \in S(r+1) \cup S(r-1)$ that is adjacent to $S$ and "surrounds" it. Let $r \in \mathbb{Z}$ be such that $S(r)$ is nonempty. Let $S_{0} \in S(r)$. Inductively, define $S_{n+1}$ to be $S_{n}$ if $S_{n}$ is infinite, and if not, let $S_{n+1}$ be some $S \in S *$ which surrounds $S_{n}$. Clearly, $\left|S_{n}\right| \geq n$. Hence $g$ is not semi-regular.

Note: This also shows that there is no regular map

$$
\left.\mathbb{Z}^{2} \rightarrow \text { reg Lamplighter(Lamplighter }(\mathbb{Z})\right)
$$

There is a more general way to see the proposition, which we will sketch in a later remark.

Before Question 5.2, maybe consider

Question 6.2. Does $\mathbb{H}^{2} \rightarrow_{\text {s-reg }} T \times \mathbb{Z}$ ?

Gromov [14] defined the notion of asymptotic dimension of a metric space, which is defined as follows. Say that $X$ has asymptotic dimension at most $D$ if for every $s>0$ there is a partition (i.e., coloring) $X=Y_{1} \cup Y_{2} \cup \cdots \cup Y_{D+1}$ such that each $Y_{j}$ can be partitioned into a collection of pieces of bounded diameter (depending only on $s$ ), with the distance between any two of the pieces at least $s$. See [3] for a nice survey on the asymptotic dimension.

Now, if $X$ maps semi-regularly into $\mathbb{Z}^{d}$, then the asymptotic dimension of $X$ is bounded from above by the asymptotic dimension of $\mathbb{Z}^{d}$ (which is $d$, [3]). To see this, just pull back the colorings in the definition of asymptotic dimension using the semi-regular map. By a similar argument one can show that there is no (semi-)regular map from $T \times T \times T$ to $T \times T$ (because the asymptotic dimension of the former is 3 , and that of the latter is 2), and that there is no (semi-)regular map from $\mathbb{R}^{d}(d \geq 3)$ to $T \times T$ (even though the separation of $T \times T$ is larger).

The question is whether this observation captures the partial order among "good" classes of spaces. To better understand the partial order given by semi-regular embeddings, the following is a relevant question.

Question 6.3. Suppose that $G$ is a transitive (homogeneous) locally finite graph with asymptotic dimension $d$. Does it follow that $\mathbb{Z}^{d} \rightarrow_{\text {s-reg }} G$ and $G \rightarrow_{\text {s-reg }} \mathbb{Z}^{d}$ ? 
Remark. We have just mentioned that the asymptotic dimension is monotone increasing under semi-regular maps. Hence there is no semi-regular map from $\mathbb{Z}^{n}$ to $\mathbb{Z}^{m}$ when $n>m$, giving an alternative proof for the above proposition.

Remark. Later Oded showed that $\mathbb{H}^{2}$ and $\mathbb{R}^{2}$ are semi-regularly equivalent. The proof is unfortunately lost.

Acknowledgment. The first author was supported by the Renee and Jay Weiss Professorial Chair of Theoretical Mathematics, and the last author by Swiss NSF grants PP0022-118946, Sinergia grant CRSI22-130435 and MTA Renyi "Lendulet" Groups and Graphs Research Group.

Thanks to Gil Kalai, Mike Freedman and Yehuda Shalom for useful discussions. Thanks to David Wilson for help with locating old emails and notes by Oded. Thanks to Sasha Sodin for his useful comments on a previous version. We are grateful to Tatiana Smirnova-Nagnibeda and an anonymous referee for valuable suggestions.

\section{References}

[1] N. Alon, P. Seymour, and R. Thomas, Planar separators. SIAM J. Discrete Math. 7 (1994), 184-193. Zbl 0797.05039 MR 1271990

[2] N. Alon, P. Seymour, and R. Thomas, A separator theorem for nonplanar graphs. J. Amer. Math. Soc. 3 (1990), 801-808. Zbl 0747.05051 MR 1065053

[3] G. Bell and A. Dranishnikov, Asymptotic dimension. Topology Appl. 155 (2008), 1265-1296. Zbl 1149.54017 MR 2423966

[4] I. Benjamini and G. Kozma, Nonamenable Liouville graphs. Preprint 2010. arXiv: 1010.3365

[5] I. Benjamini and O. Schramm, Harmonic functions on planar and almost planar graphs and manifolds, via circle packings. Invent. Math. 126 (1996), 565-587. Zbl 0868.31008 MR 1419007

[6] I. Benjamini and O. Schramm, Lack of sphere packing of graphs via non-linear potential theory. Preprint 2009. arXiv:0910.3071

[7] H. L. Bodlaender, A partial $k$-arboretum of graphs with bounded treewidth. Theoret. Comput. Sci. 209 (1998), 1-45. Zbl 0912.68148 MR 1647486

[8] H. L. Bodlaender and J. Engelfriet, Domino treewidth (extended abstract). In Graphtheoretic concepts in computer science (Herrsching, 1994), Lecture Notes in Comput. Sci. 903, Springer, Berlin 1995, 1-13. MR 1354184

[9] S. Buyalo and V. Schroeder, Embedding of hyperbolic spaces in the product of trees. Geom. Dedicata 113 (2005), 75-93. Zbl 1090.54029 MR 2171299

[10] G. David and S. Semmes, Regular mappings between dimensions. Publ. Mat. 44 (2000), 369-417. Zbl 1041.42010 MR 1800814

[11] R. Diestel, Graph theory. Graduate Texts in Math. 173, Springer-Verlag, New York 1997. Zbl 0873.05001 MR 1448665 
[12] É. Ghys, A. Haefliger and A. Verjovsky (eds.), Group theory from a geometrical viewpoint. World Scientific Publishing Singapore 1991. Zbl 0809.00017 MR 1170362

[13] M. Gromov, Hyperbolic groups. In Essays in group theory, Math. Sci. Res. Inst. Publ. 8, Springer-Verlag, New York 1987, 75-263. Zbl 0634.20015 MR 0919829

[14] M. Gromov, Geometric group theory (Sussex, 1991), vol. 2: Asymptotic invariants of infinite groups. London Math. Soc. Lecture Note Ser. 182, Cambridge University Press, Cambridge 1993. Zbl 0841.20039 MR 1253544

[15] R. Krauthgamer and J. R. Lee, The intrinsic dimensionality of graphs. In Proceedings of the Thirty-Fifth Annual ACM Symposium on Theory of Computing (STOC 2003), ACM Press, New York 2003, 438-447. Zbl 1192.05039 MR 2120459

[16] R. J. Lipton and R. E. Tarjan, A separator theorem for planar graphs. SIAM J. Appl. Math. 36 (1979), 177-189. Zbl 0432.05022 MR 524495

[17] G. L. Miller, S.-H. Teng, W. Thurston, and S. A. Vavasis, Separators for sphere-packings and nearest neighbor graphs. J. ACM 44 (1997), 1-29. Zbl 0883.68100 MR 1438463

[18] G. L. Miller, S.-H. Teng, W. Thurston, and S. A. Vavasis, Geometric separators for finiteelement meshes. SIAM J. Sci. Comput. 19(1998), 364-386. Zbl 0914.65123 MR 1618871

[19] V. Nekrashevych, Self-similar groups. Math. Surveys Monogr. 117, Amer. Math. Soc., Providence, RI, 2005. Zbl 1087.20032 MR 2162164

[20] N. Robertson and P. D. Seymour, Graph minors. V. Excluding a planar graph. J. Combin. Theory Ser. B 41 (1986), 92-114. Zbl 0598.05055 MR 854606

Received July 28, 2009; revised August 29, 2011

I. Benjamini, Faculty of Mathematics and Computer Science, The Weizmann Institute of Science, P.O. Box 26, Rehovot 76100, Israel

E-mail: Itai.Benjamini@weizmann.ac.il

Á. Timár, Fakultät für Mathematik, Universität Wien, Nordbergstrasse 15, 1090 Wien, Austria

E-mail: adam.timar@hausdorff-center.uni-bonn.de 